\title{
Sir Joseph John Thomson, O.M., D.Sc., LL.D., F.R.S.
}

Joseph John THOMSON * was the son of a Manchester bookseller, and was born in that city on December I8, 1856. At the age of fourteen he entered Owens College (now the University of Manchester), and four years later won an entrance scholarship in mathematics at Trinity College, Cambridge, where he read for the Mathematical Tripos. In I880 he graduated as Second Wrangler, and at once set to work on the preparation of a thesis for the Fellowship competition of the College.

His starting-point was the known theorem of dynamics that if two bodies, $A$ and $B$, influence each other's motion, say by attraction, and if we observe the motion of $B$ which results when $A$ moves in a definite way, then by dynamical theory we can deduce the motion of $A$ when that of $B$ is specified, even though we do not know the precise nature of the connection between them. Believing that all physical phenomena could be explained by dynamical principles, Thomson tried to extend this theorem to the more general case, which occurs in Physics and Chemistry, when instead of two bodies attracting each other we have two phenomena, $\mathrm{A}$ and $\mathrm{B}$, which mutually influence each other. We may be ignorant of the details of the molecular mechanism connecting the phenomena, but, as Thomson showed, we may by general dynamical theory be able to deduce the effect of $A$ on $B$ when we have observed the effect of $B$ on $A$. Thus, if we observe that the coefficient of magnetisation of a piece of soft iron decreases as the temperature increases, then we can infer that a magnet will become heated when it is moved from weak to strong parts of the magnetic field. The dissertation containing these results won a Fellowship at the first attempt, and the dominant idea in it has had a great influence on the subsequent development of physical chemistry.

It was on the border-line between chemistry and physics that Thomson found the subject which he next attacked, and which by a series of brilliant discoveries he ultimately solved, namely, the conduction of electricity through gases. His starting-point here was Lord Kelvin's representation of the atoms of matter by vortex-rings in a perfect fluid. In I 883 Thomson constructed a model of the electric discharge through gases by representing the atoms of a gas as vortex-rings: the combination of two atoms to form

* This obituary notice is extracted from the President's Address delivered at the Annual Statutory Meeting of the Society on October 28, I940. 
a molecule would then correspond to the union or pairing of two vortexrings, a phenomenon which was well known experimentally and which he had investigated mathematically. Now suppose that there is a quantity of gas in an electric field. The electric field he represented by a distribution of velocity in the medium whose vortex-motion constitutes the atoms of the gas: the disturbance due to this distribution of velocity will cause some of the paired vortex-rings to dissociate, and so a mathematical theory of the phenomenon of conduction in gases becomes possible. He worked out the conditions for the disruptive discharge, and showed that the intensity of the field necessary to produce discharge should diminish when the gas is rarefied.

To the question of conduction in rarefied gases he now devoted his attention, being moved by the consideration that the properties and laws of gases are simpler than those of solids and liquids, and that the kinetic theory enables us to form clearer mental pictures of what goes on in a highly rarefied gas. It had been shown in 1869 by Hittorf that the phosphorescent light in the neighbourhood of the cathode in a dischargetube consists of a disturbance of some kind which is propagated in straight lines from the cathode: for when a solid body is placed in the tube it casts a shadow. This disturbance thus came to be known as the cathode rays: and a lively discussion went on for over twenty years as to whether these rays were, like rays of light, a disturbance in the æther, or whether they consisted of a torrent of small charged particles torn off the cathode. Most of the German physicists, especially Hertz and Lenard, held to the æther hypothesis, while the British, particularly Varley, Crookes, FitzGerald, and Thomson, advocated the projectile theory. Hertz supported his thesis by showing experimentally that the rays did not appear to produce any external electric or magnetic field, or to be deflected by an electrostatic field, and, above all, that they were capable of passing through films of metal which were so thick as to be quite opaque to ordinary light: it seemed inconceivable that particles of matter should not be stopped by even the thinnest gold-leaf. (At that time the alpha rays of radium, which are undoubtedly streams of particles and have a remarkable penetrating power, were not as yet discovered.) On the other hand, the upholders of the particle theory showed that the cathode rays are deflected by a magnetic field: and in 1894 Thomson, by means of a rotating mirror, succeeded in measuring their velocity, obtaining a value $\left(\mathrm{I} \cdot 9 \times 10^{7} \mathrm{~cm}\right.$. per sec.) so much smaller than the velocity of light that it seemed scarcely possible to conceive of the rays as vibrations of the æther. A further blow was dealt at the æther hypothesis in the following year, when Perrin, having received the rays in a metallic cylinder placed in front of the cathode, found that 
the cylinder became charged with negative electricity. Thomson then extended this experiment by showing that when the rays were deviated by a magnet they still communicated a charge to any vessel placed to receive them. This appeared to demonstrate conclusively that they transported negative electricity, and therefore were, presumably, particles of matter.

It seemed probable that if the charged-particle theory were true, the charges of all the particles would be equal, and would in fact each be equal to an elementary quantum or atom of electricity, which would not be capable of further subdivision. Thus the question as to whether electricity was continuous, or existed only in discrete and identical units, was involved in the discussion. The theory of electric displacement, which had been put forward by Maxwell, favoured the concept of electricity as a continuum: and Maxwell's theory had by this time won general acceptance, chiefly as a result of Hertz's experimental demonstration of electric waves. On the other hand, the facts of electrolysis seemed to show that an absolute unit of electric charge exists, namely, that amount of it which attends each chemical bond or valency: and in fact the magnitude of the charge carried by the hydrogen atom in electrolysis was well known, the charges carried by other atoms being either equal to this or (positive or negative) multiples of it.

In 1896 and 1897 Thomson, by a brilliant series of experiments, succeeded in removing all the objections to the charged-particle theory of the cathode rays, and finally determined their actual nature. $\mathrm{He}$ first dealt with Hertz's failure to deflect the rays by an electrostatic field; by carrying the exhaustion to a much higher degree, he removed the effects of the ionisation of the residual gas in the tube, which, as he showed, had been the cause of Hertz's negative result, and thus he obtained the expected deflection. He now subjected the rays to an electric and a magnetic field simultaneously, and adjusted the fields until the deflections in opposite directions balanced each other: this experiment, as he showed, provided data for determining the velocity of the particles. Then, by measurements of the deflection by a magnetic field alone, he obtained the value of $\frac{m v}{e}$, where $m$ denotes the mass of one of the particles, $v$ its velocity, and $e$ its electric charge. Since $v$ was already known, he was able to calculate $(m / e)$, the ratio of the mass of a cathode-ray particle to the charge which it carries. This ratio was found to be independent of the nature of the rarefied gas or of the cathode: and, to Thomson's great surprise, it proved to be less than the thousandth part of the value of $(m / e)$ for the hydrogen ion in electrolysis. If the charge were the same as that of the hydrogen 
ion-and by use of a brilliant discovery of C. T. R. Wilson he proved this to be the case-then there was no escape from the conclusion that the particle whose mass was thus measured was very much smaller than the smallest atom.

The first public announcement of this great discovery was made by Thomson in an Evening Discourse at the Royal Institution on April 30, I 897. "The assumption of a state of matter more finely subdivided than the atom of an element," he said, "is a somewhat startling one: but a hypothesis that would involve somewhat similar consequences-viz. that the so-called elements are compounds of some primordial element-has been put forward from time to time by various chemists."

In order to explain this last remark, it must be recalled that from the very beginnings of physics, amongst the Milesian philosophers in the sixth century B.C., the doetrine had been maintained that all things are but different forms of one single primary and universal substance. Thales conjectured that it might be water, Anaximenes that it might be air, and Anaximander that it was a boundless indefinite something- out of which everything is generated and to which everything returns. Modern science has entertained the same idea, in many different forms: as we have seen, Lord Kelvin suggested that all atoms might be vortexrings in an infinite liquid, which would be precisely Anaximander's

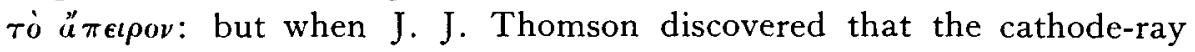
particles were the same, whatever atoms they were torn out of, his mind recurred chiefly to the hypothesis put forward in 18 I 5 by Prout, namely, that the atomic weights of the elements are whole-number multiples of the atomic weight of hydrogen, and that, in fact, hydrogen is the universal primordial substance. Thomson now conjectured that Prout had been wrong in selecting hydrogen, and that the fundamental place in nature belonged rightly to the newly discovered cathode-ray particles, or "corpuscles" as he called them, which were very much smaller than the hydrogen atom and were now proved to be contained in the atoms of all elements. This idea seems to have fascinated him at the time, as well it might.

Later researches-most of them carried out or inspired by Thomson himself-have shown the necessity of modifying this opinion as to the place of the cathode-ray corpuscles in the scheme of the universe. Since they carry charges of negative electricity, while the atom as a whole is neutral in respect of charge, it follows that some part of the atom must be positively charged. To investigate this, Thomson examined another set of rays obtained in the vacuum discharge. More than a decade previously, Goldstein had shown that when the cathode of a discharge-tube is 
perforated, radiation of a certain type passes through the perforations into the part of the tube behind the cathode, travelling in the direction opposite to the cathode rays: to this radiation he had given the name canal rays. Shortly after Thomson's work on the cathode rays, it was shown that the canal rays are torrents of positively charged particles, the value of $(\mathrm{m} / \mathrm{e})$ being immensely larger than that obtained for the cathode rays, and indeed of the same order of magnitude as the corresponding ratio in electrolysis. In 1907 Thomson made an extensive experimental study of these canal rays, or positive rays as he now called them, determining the velocity and the ratio $(m / e)$ by shooting the rays through a narrow tube, so as to obtain a small spot on a phosphorescent screen or a photographic plate, and subjecting them between the tube and the screen to an electric field and also a magnetic field, so as to deflect the beam of particles. $\mathrm{He}$ showed that all particles having the same value for $(m / e)$ would be spread out by the two fields so as to strike the screen in points lying on a parabola; thus, particles of different mass would give different parabolas. Parabolas were found corresponding to the atoms and molecules of various gases in the discharge-tube: and the atomic weights of the particles could be at once inferred from measures of the parabolas. By applying this method of positive-ray analysis to the gas neon (since used in the familiar advertisement lights) he found, in addition to a parabola belonging to atomic weight 20 , another corresponding to atomic weight 22 . These proved to be, both of them, atoms of neon, but of different masses: Thomson had in fact discovered the first example of two atoms having the same chemical behaviour but different physical characteristics: as we now say, two isotopes of the same chemical element.

The innumerable investigations that have been carried out in atomic physics since the beginning of the present century have not so far led up to any universal primordial substance such as Thomson thought he had found in 1897 . We have progressed somewhat in its direction, for we now know that the atoms of the 92 chemical elements are all compounded of a much smaller number of more fundamental particles. Of these, the first is Thomson's cathode-ray corpuscle, now always known as the electron: it is not, however, the only elementary particle: there are others-the proton, the neutron, the positron, the light-quant, the meson, and perhaps the neutrino. Some of these can certainly be generated from the others: and it seems probable that every one of them can be created or annihilated, so that the doctrine of permanent indestructible ultimate particles, as taught by Leukippus and Democritus, is definitely false: but more than this we cannot, in the present state of knowledge, affirm.

The discovery of the electron established Thomson's reputation as 
the first of living experimental physicists: and it was in this aspect that he was generally regarded during the latter half of his life. But his contributions to theoretical or mathematical physics were scarcely less important than the fruits of his researches in the laboratory. Perhaps his greatest achievement in pure theory was the discovery (in I 893) of electromagnetic momentum; namely, that in an electromagnetic field there is stored in every unit volume an amount of mechanical momentum, proportional to the vector-product of the electric and magnetic vectors. This principle is necessary for the construction of the energy-tensor; and without it, neither relativity-theory nor quantum-electrodynamics could have developed.

During the whole of his tenure of the Cavendish chair Thomson was the active head and inspirer of a great research school. The institution of the status of "advanced student" at Cambridge in I895 led to a great increase in the numbers of young graduates of other universities who came to work in his laboratory, with the consequence that to-day nearly all the important chairs of Physics in the Empire are filled by his disciples.

In 19I 8 he was elected Master of the great foundation of which he had been a member uninterruptedly since $\mathrm{I} 875$.

"How fortunate I have been throughout my life!" he wrote, near the end of it. "I have had good parents, good teachers, good colleagues, good pupils, good friends, great opportunities, good luck, and good health." He lived to be eighty-three, dying at Trinity Lodge on August 30,1940 , and was buried on September 4 in Westminster Abbey.

He was elected an Honorary Fellow in 1905.

E. T. W. 